\title{
Promising Prospects in Mobile Search: Business as Usual or Techno-Economic Disruptions?
}

I

t has taken more than a decade of intense technical and market developments for mobile Internet to take off as a mass phenomenon. And it has arrived with great intensity: an avalanche of mobile content and applications is now overrunning us. Similar to its wired counterpart, wireless Web users will continuously demand access to data and content in an efficient and userfriendly manner. If the lessons of the fixed Internet experience hold, then search engines will also become pivotal in the mobile environment from both a technological and commercial perspective. From the technological point of view, as a key platform shaping the interfaces of other applications and content, and, from a commercial point of view, as one of the most notorious candidates for a prosperous business case. Mobile search is, thus, becoming an increasingly important market area and topic for research.
Following promising market prospects, many actors are seeking to develop the next big search application. Nevertheless, how to gain advantages from existing (and future) mobile technologies and where to position themselves to enjoy sufficient revenues are far from obvious. A major reason is that search applications are neither simple nor autonomous building blocks. Rather, search functionalities are tightly embedded into the value chain of wider mobile services, which themselves can be numerous and of diverse natures. In techno-economic terms, we could portray the search functionality as a key constituent in an ecosystem where industrial players compete and/ or collaborate to generate successful and scalable business strategies in a highly dynamic and still-emerging market landscape. The purpose of this article is, therefore, to take a look at the economic side of mobile search without losing sight of the influence of technology.

\section{WHICH SEARCH} FOR MOBILE SEARCH?

Applications on mobile devices relying upon search technology can be grouped into the following two cases:

Case A: Those that adapt or emulate existing Web search services to the mobile environment.

- Case B: Those that also exploit the unique features of mobile devices or the environment in which they operate.

In the first search-as-usual case (Case A), mobile search applications evolve by migrating established personal computer (PC)-based Internet search tools to a mobile environment; possibly complementing it with some mobility enhancement function such as refining the results taking into account the user's location. Such migration can pose severe technical challenges, mainly on enhancing the efficiency to retrieve relevant content in all digital formats, particularly in an audiovisual context, and on responding as accurately as possible to natural language queries. 
In the future, mobile search will increasingly (Case B) become more sensitive to a logic that is driven fundamentally by personalized and context-based services offering a search experience well beyond of today's wired Internet world [1]. Mobile search will make effective use of contextual information (relevant data embedded in the mobile device, information in the surrounding environment, and users' profiles or behavioral patterns) to improve the relevance of search results and/or to provide a more valuable and entertaining user experience as the "engine within" new types of applications.

In both Cases A and B, techno-economic factors pushing mobile Internet are obvious direct enablers. These include the (still) growing mobile penetration, increasing mobile broadband availability and affordability, improvement in usability and affordability of smartphones, and availability of useful mobile content and applications. Obviously, the more mobile Internet grows, the more mobile search will also intensify, but the real challenge is how to monetize it. Advertising seems the intuitive response when comparing with current Internet search, particularly for Case A mobile search applications. For Case B applications, two factors will influence the business model: 1) the feasibility to monetize the added value provided by mobile search within a given applications and 2) the economic value of the search functionality with regard to the totality of the value chain of the service. It is the combination of these two elements that would determine the sustainability of new types of mobile search ventures. So far, no absolute statements can be given, but we can grasp some of its potential by analyzing the position and weight of mobile search within the upcoming mobile ecosystem.

\section{THE MOBILE SEARCH ECOSYSTEM}

We use the ecosystem metaphor to illustrate the relationship of crucial, interacting players within a given market environment in which none of players controls the system completely. The ecosystem was originally proposed as a gen- eral model to depict relationships for any type of mobile content or application [2] and can be adapted to the mobile search case. Figure 1 shows the three-layered structure, typical of information and communications technology (ICT) ecosystems [3], and includes the four steps in mobile search evolution (light to dark blue underlying boxes, counterclockwise): the initial and simple on-portal approach (left), the subsequent ondevice and additional input functionalities (down), the current mobile version of Web search (right) and, finally, the future context-aware search (up).

Within the ecosystem dynamics some players will aim at, and eventually succeed in, integrating as many activities as possible for a more effective control of the value network. This approach is resulting in a platformization of the ecosystem in which main players strive to pull together (in a loose or tight cooperative scheme) all the necessary functions to provide mobile offerings on a common set of hardware, software, and technoeconomic specifications. The aforementioned scheme was used until recently by mobile carriers to control a plurality of the elements within the value chain. This scheme, also known as walled garden, silo model, or on-portal model [4], has increasingly come under pressure. Technological progress, together with an increasing user demand for an unrestricted and wide choice of content and applications, have caused profound changes in the mobile industry structure [5]. Other players have come to the forefront with considerable strength. In a very short time, device suppliers succeeded in creating alternative platforms where users are guided to stay as much as possible within a single value structure. Apple or Google's supported Android are currently the prominent examples [6], although others (Nokia, RIM, Microsoft, even mobile carriers themselves) have not renounced to maintain or improve their privileged positions.

Mobile search providers have something to say in this battle for control of the value in the ecosystem. In the $\mathrm{PC}$ environment, Web search engines enjoy a unique position as a point of entry for end users to retrieve, subscribe, and use content and applications. Provided they succeed in producing the necessary adaptations to the mobile environment, steering mobile platforms or connecting them to their existing portfolio of services in the wired Internet is their ultimate goal. The Google example shows how elements fit together. Google provides 1) Android as an open operating system basically (but not only) for mobile devices, 2) a set of software facilities for developers on this platform, 3) an application storefront (and the corresponding processes for charging, billing, and sharing revenues with developers and publishers), and 4) mobile devices supplied by an increasing number of manufacturers.

And future prospects are even more promising. Potential disruptions are expected to arise from leveraging value in context and personal profile while keeping connected with the social network. Mobiles will gradually turn into the natural device to bridge the physical world surrounding us with the wealth of information on the net, whereby search engines operate in the background to provide the link between both worlds.

From the modeling perspective, understanding and monitoring the interrelations of applications within the ecosystem are necessary to determine what the opportunities for new techno-economic models are. Given its dynamics, an eventual breakthrough, for instance, of context-aware services (and search) will reshape the borders of the mobile ecosystem, offering new opportunities for new and existing players, and modifying the current balance among mobile platforms.

\section{BUSINESS MODELS \\ FOR MOBILE SEARCH}

Classification schemes for mobile search may be based on the reach of the search (on-device search, on-portal search, offportal search, social network-based search), based on the method used to perform the search (Web-based mobile engines, messaging service-based, voicebased search engines), or based on the 
features added to the search (conventional text-, multimedia-, semantic-, context-aware search). Irrespective of the technological or typological characteristics, there are no conceptual differences with regard to the feasibility of business models. Indeed, there are only four major sources of revenues for mobile search companies.

\section{ADVERTISING}

Following the success in the wired Internet, advertising is the most obvious candidate for a business model also in mobile search. Search results are provided free of charge to final users and revenues are generated from third-party advertisers. Advertising models come in various facets and business tactics. The most common is the inclusion of sponsored links on search results, frequently including a direct response method as well (a link to a microsite, a click-to-call link, or a short code). Other options are keyword bidding, especially for marketers offering digital content, or off-portal campaigns for specific services, such as travel, restaurants, automotive, or consumer electronics. Likewise, ad campaigns for products related to what mobile operators offer on their mobile portals (ringtones, games, wallpapers, music, and video) is a typical example of on-portal search.

Advertising is considered by many as the cash cow in the deployment of advanced mobile applications [7]. But evidence on how exactly the revenue stream will be generated is still unclear, rather it seems that many firms bet on advertising only because alternative concepts to render mobile applications profitable are even more vague. In spite of the diffuse picture advertisers perceive that business-as-usual ads are not going to deliver the expected impact.
Therefore, the focus is shifting towards more sophisticated approaches, like targeted product placement or dire personalization, which also offers a better approach to an increasingly fragmented audience across media.

Another relevant trend is advertising within the mobile application itself, rather than guiding users via the browser to sponsored links. This approach (rich media advertising) would allow the inclusion of search capabilities into a number of mobile applications while keeping the advertising business model.

\section{PRODUCT OR SERVICE TO FINAL USERS}

Generating revenues from final users must always be based upon the premise that the value offered is attractive enough for users to pay for the search service. The following business models respond to this criterion:

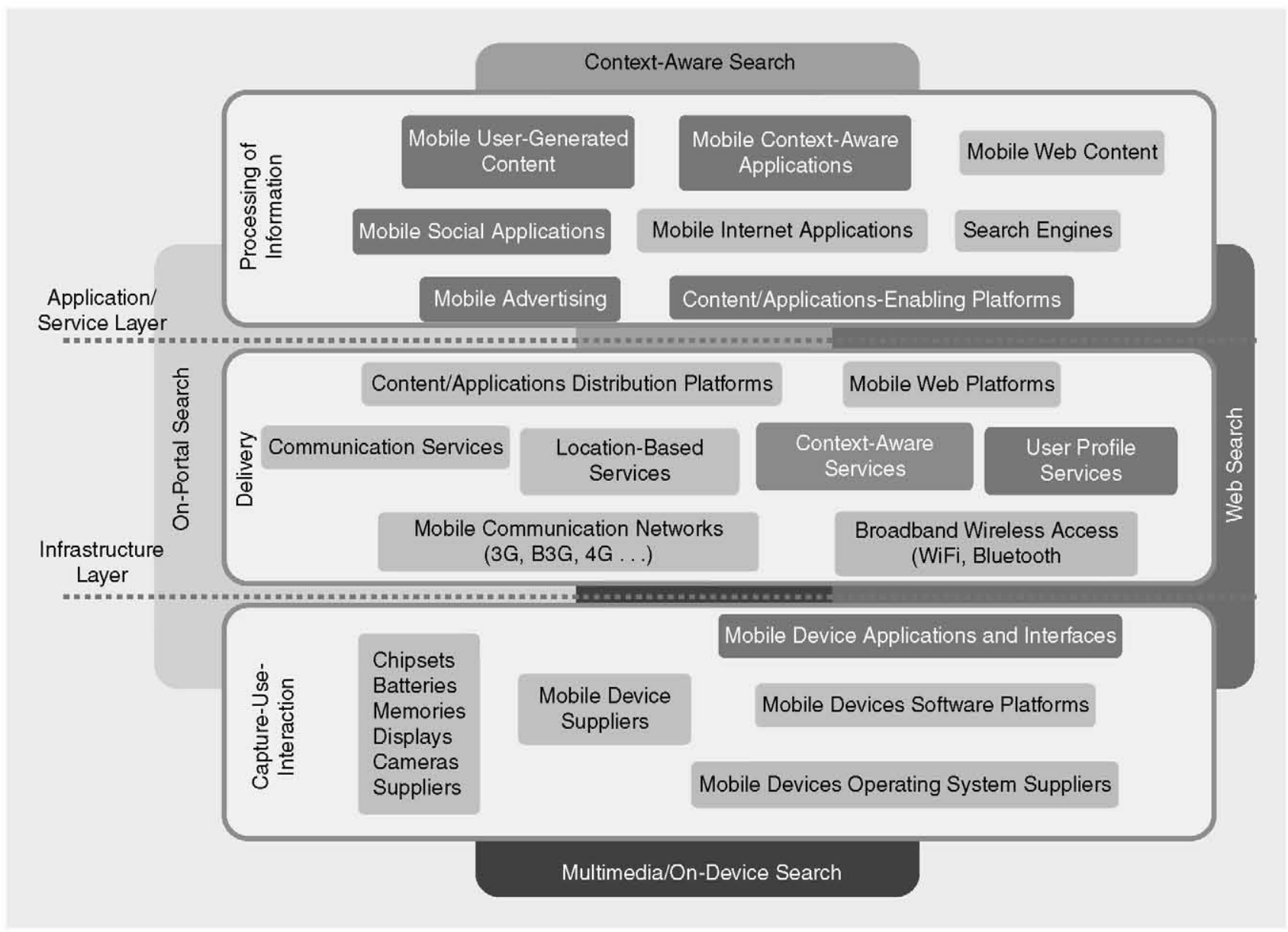

[FIG1] Activities and players in the mobile search ecosystem (adapted from [2]). 
- Pay as you go (pay as you need) are based on impulse purchase when there is a high need or urgency for particular search results (examples include necessary search services in unknown environments).

Premium services provide more value than its free basic functionality (this added value could be in the interface, the range of inputs and/or results, or in additional information provided in the results to the query, for instance, a telephone number ready to be called).

- Value-added services offer an improved version requiring an additional contract for particular services, like niche applications or professional services (for instance, search of audiovisual content from an image taken from a mobile device in a journal context).

- Subscription models are when the search service is supplied for a specific period of time (a month, a year) or in specific circumstances (travel, a city).

Due to the success of application stores, business models that could step on their particular features are also popular. Beyond the traditional models above, value-added applications, i.e., applications downloaded from an application store and from which new functionalities can be accessed, may incorporate timebased billing for subscriptions to services, event-based billing, or even item-based billing (e.g., payment as a function of the usefulness of the results of a query).

\section{PACKAGED WITH SOME OTHER PRODUCT OR SERVICE}

This business model appears when search applications are considered as just an additional commodity for other products and services; its usefulness of search being perceived as low added value. In this case, the revenue for search-related parties would be basically limited to developing and licensing white label search applications or a specific search application to be embedded in other services.

Typical scenarios are packaging the search application with the mobile device for suppliers or telecom operator services (for on-portal or on-device search) or with a nonmobile product or service (travel, for instance). As search would merely constitute an add-on to existing products or services, users would unlikely be willing to pay separately for search functionalities, rather they would be charged for the complete package. Autonomous revenues obtained through search (through

\section{r DUE TO THE SUCCESS OF APPLICATION STORES, BUSINESS MODELS THAT COULD STEP ON THEIR PARTICULAR FEATURES ARE ALSO POPULAR.}

any of the business models previously considered) would typically go to the outsourcing player rather than the outsourced search developer.

\section{INDIRECT REVENUES}

In addition, complementary business models to the above could be considered, the most important being those exploiting user profile derived from the queries and results for marketing purposes. Their implementation is delicate given legal restrictions and growing privacy concerns by policy makers and users. Anonymizing data to prevent tracing back to individuals and groups is technically viable nevertheless the value of information diminishes as it gets more aggregated. Striking the right balance between privacy, personalization services, and sustainable business model remains a foremost challenge.

Other commercial options for indirect revenues of less significance include merchandising and affiliation. Merchandising would relate mobile search with product or service sale (e.g., a musical search application would sell music or merchandising from artists), while affiliation allows the search provider to obtain revenues whenever a user accesses a service or purchases a product (a book, for instance) from the retrieved result search.
Nonprofit search applications can also contribute to the viability of mobile search. Examples include the use of search in noncommercial public services, such as a city council providing a mobile search service for transportation schedules, or search applications maintained by a users community (typically free for final users).

\section{DÉJÀ-VU OR SURPRISES?}

While it was just in its infancy a short time ago, mobile Internet is expanding rapidly and steadily into a growth market and with it also mobile search, as the few available market data indicate. And what are the prospects of mobile search? Is mobile search just following the same steps as in the case of wired Internet, or should we expect major disruptions, technically and economically?

In the short term, search-as-usual is the first model for companies to kickoff with and responds to the commonly accepted view that advertising is also likely to be the predominant one for mobile search. The spin received from the $\mathrm{PC}$ environment give established players time to first take up a big part of the market and, then, evolve smoothly into a new and smarter search if needed. In this first-mover advantage scenario, advertising seems the de facto business model to move forward. Traditional advertising strategies and formats need to be (and are currently being) adapted to the mobile environment [8], paying attention so inherent conflicts among the interested parties can be solved [9].

In the medium term, search should be fully adapted to the particular circumstances of the user. As mobile Internet will keep growing, the combination of context awareness with relevant, useful, and interesting context-related information will make the difference in mobile search. In this scenario, some of the possibilities remain commercially unexplored, while in some other cases, companies are still in the phase of evaluating user responses to propose new services and, more importantly, still experimenting with how exactly to monetize these services before embarking on large-scale 
deployments. It is unclear which of the many variants of this increasingly personalized model will be successful. Revenue models built around a premium service, a value-added service, impulse purchases, or a subscription service are likely to survive only in niche applications that exploit particular location environments to satisfy very specific user needs.

What will happen in the long term? In a visionary scenario, search engines would evolve into fully fledged recommendation engines able to update user preferences automatically in real time, including social network and use patterns. Anticipatory functions, ranging from serendipity content discovery, to learning and entertainment, would be possible. Such applications would require a higher degree of user profiling and behavioral tracking than we are currently accustomed. This causes a dilemma. On the one hand, the lack of personalized data limits the value of useful applications. On the other hand, advanced personalized services require extended access to personal data provoking privacy concerns. A privacy backlash could prejudice advanced mobile search. Thus, ensuring privacy by law enforcement, by techno- logical design, and/or by user choice are all necessary elements to pursue.

Who will be the winners in this race? Technology enables much change but it is the social force of users that changes the role of mobile Internet in our society. Mobile search is no exception, and its value must be scrutinized on its value and compatibility with the modern lifestyle, rather than its technological capacity. Thus, whoever is able to follow the logic of mobile search developing applications that would match the increasingly mobile, global, connected, and individualized users' necessities would get a foothold in the market place.

\section{ACKNOWLEDGMENT}

The opinions expressed in this article are those of the authors and do not necessarily reflect the views of the European Commission.

\section{AUTHORS}

José Luis Gómez-Barroso (jlgomez@cee. uned.es) is a professor at Universidad Nacional de Educación a Distancia in Spain.

Claudio Feijoo (cfeijoo@cedint.upm. es) is a professor at Universidad Politécnica de Madrid in Spain.
Ramón Compañó (ramon.compano@ec.europa.eu) is the program manager of the Institute for Prospective Technological Studies (Joint Research Centre of the European Commission).

\section{REFERENCES}

[1] O. Westlund, J. L. Gómez-Barroso, R. Compañó, and C. Feijóo, "Exploring the logic of mobile search," Behav. Inform. Technol, to be published. .

[2] C. Feijóo, I. Maghiros, F. Abadie, and J. L. Gómez-Barroso, "Exploring a heterogeneous and fragmented digital ecosystem: Mobile content," Telemat. Inform. vol. 26 , no. 3 , pp. 282-292, 2009.

[3] M. Fransman, The New ICT Ecosystem. Implications for Europe. Edinburgh: Kokoro, 2007.

[4] S. Ramos, C. Feijóo, and J. L. Gómez-Barroso, "Next generation mobile network deployment strategies," J. Inst. Telecommun. Prof., vol. 3, no. 1, pp. $13-19,2009$

[5] J. West and M. Mace, "Browsing as the killer app: Explaining the rapid success of Apple's iPhone," Telecommun. Policy, vol. 34, no. 5-6, pp. 270-286, 2010.

[6] G. Goggin, "Adapting the mobile phone: The iPhone and its consumption," Continuum, vol. 23, no. 2, pp. 231-244, 2009.

[7] M Leppaniemi and H. Karjaluoto, "Factors influencing consumers' willingness to accept mobile advertising: a conceptual model," Int. J. Mobile Commun., vol. 3, no. 3, pp. 197-213, 2005.

[8] T. Park, R. Shenoy, and G. Salvendy, "Effective advertising on mobile phones: A literature review and presentation of results from 53 case studies," Behav. Inform. Technol., vol. 27 , no. 5, pp. 355-373, 2008.

[9] R. Wilken and J. Sinclair, "Waiting for the kiss of life: Mobile media and advertising," Convergence, vol. 15 , no. 4 , pp. $427-445,2009$. 